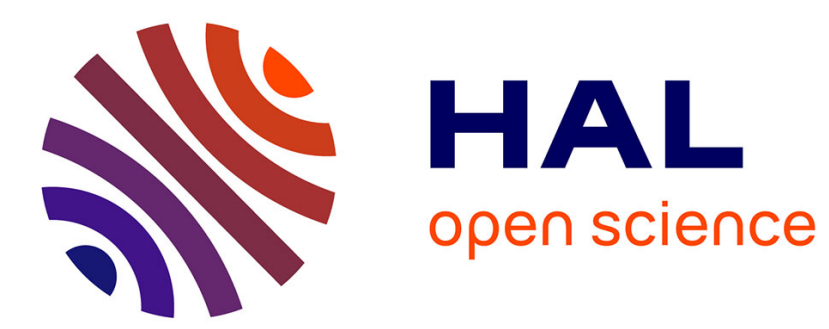

\title{
Le fantasme du secret d'État autour du 17 octobre 1961
}

Sylvie Thénault

\section{To cite this version:}

Sylvie Thénault. Le fantasme du secret d'État autour du 17 octobre 1961. Matériaux pour l'histoire de notre temps, 2000, Le secret en histoire, 58, pp.70-76. 10.3406/mat.2000.404254 . hal-02355865

\section{HAL Id: hal-02355865 \\ https://hal.science/hal-02355865}

Submitted on 8 Nov 2019

HAL is a multi-disciplinary open access archive for the deposit and dissemination of scientific research documents, whether they are published or not. The documents may come from teaching and research institutions in France or abroad, or from public or private research centers.
L'archive ouverte pluridisciplinaire HAL, est destinée au dépôt et à la diffusion de documents scientifiques de niveau recherche, publiés ou non, émanant des établissements d'enseignement et de recherche français ou étrangers, des laboratoires publics ou privés.

\section{(이)(\$)}

Distributed under a Creative Commons Attribution - NonCommercial - NoDerivatives| 4.0 


\section{Le fantasme du secret d'Etat autour du 17 octobre 1961}

Madame Sylvie Thenault

\section{Citer ce document / Cite this document :}

Thenault Sylvie. Le fantasme du secret d'Etat autour du 17 octobre 1961. In: Matériaux pour l'histoire de notre temps, n58, 2000. Le secret en histoire. pp. 70-76;

doi : https://doi.org/10.3406/mat.2000.404254

https://www.persee.fr/doc/mat_0769-3206_2000_num_58_1_404254

Fichier pdf généré le 30/03/2018 


\section{Le fanstasme du secret autour du 17 octobre 1961}

L e 17 octobre 1961, vingt mille Algériens manifestent à Paris à l'appel du FLN, contre le couvre-feu décidé quelques jours plus tôt par le préfet de police, Maurice Papon. La répression que leur opposent les forces de l'ordre frappe les esprits par sa violence. Elle fit de très nombreux morts et blessés.

Depuis deux ans, plusieurs événements ont contribué à replacer le 17 octobre 1961 sous les feux de l'actualité. Le premier d'entre eux est le procès de Maurice Papon à Bordeaux pour complicité de crime contre l'humanité, au cours duquel a été étudié la carrière de l'accusé. Ce procès a donc été l'occasion de revenir sur le 17 octobre, notamment à travers le témoignage de Jean-Luc Einaudi, auteur de La bataille de Paris, publiée au Seuil en 1991. Par la suite, Maurice Papon a intenté à Jean-Luc Einaudi un procès en diffamation à cause d'une phrase écrite par ce dernier dans un article du Monde : "En octobre 1961, il y eut un massacre perpétré par des forces de police agissant sous les ordres de Maurice Papon " ${ }^{1}$.Cependant, le jugement rendu en février 1999 n'a pas condamné Jean-Luc Einaudi et le procureur reconnut même l'existence d'un " massacre » le soir du 17 octobre 1961.

Ces procès ont vu évoluer l'attitude des pouvoirs publics qui ont longtemps entretenu le silence sur cette question et maintenu un bilan officiel de trois morts, sans aucune crédibilité. En 1998 et 1999, deux rapports officiels ont ainsi été commandés, essentiellement dans l'objectif de recenser le contenu des archives : la commission Mandelkern a dressé l'état des archives de la police tandis que Jean Géronimi a rendu un rapport concernant les archives judiciaires ${ }^{2}$.

Enfin, en octobre 1999, est paru aux éditions Flammarion le premier livre consacré au 17 octobre rédigé à partir d'archives publiques. Sous la plume de Jean-Paul Brunet, le titre de ce livre, Police contre FLN, le drame d'octobre 1961, révèle la primauté accordée par l'auteur aux archives de la préfecture de police de Paris.

L'impression donnée par ces événements récents est que le 17 octobre reprend vie, se réveille après une longue période de coma, faite d'occultation, d'oubli, voire de camouflage. Le choix de ces termes n'a rien d'innocent car chacun, à travers sa signification, entretient un rapport spécifique avec la construction d'un secret autour de l'événement : dans le cas du camouflage, le secret est volontairement organisé, il est le résultat d'une manœuvre consciente et réfléchie; dans le cas de l'oubli, le secret est involontaire, il est la conséquence de la sélection opérée par la mémoire qui ne peut conserver le souvenir de l'ensemble des événements qui ont marqué l'actualité depuis trente ou quarante ans; l'occultation, enfin, construit inconsciemment le secret qui naît de l'effacement par la mémoire d'un événement porteur de culpabilité. Pour le 17 octobre, ces trois mécanismes sont à l'œuvre. Ils rendent la question du secret couvrant cet événement très compliquée à analyser.

Par ailleurs, pour les spécialistes de la guerre d'Algérie confrontés à l'actualité, témoins du réveil du 17 octobre dans l'opinion publique, se produit un phénomène classique : dans la mesure où ils sont informés des publications, y compris des revues universitaires à diffusion confidentielle, des travaux en cours, des programmes des séminaires de recherche, rencontres, réunions etc., un sentiment de "trop-plein " sur l'événement peut naître chez eux alors que pour le public, l'événement, méconnu, doit être révélé, sortir du secret dans lequel il est resté. La résurgence du 17 octobre s'accompagne donc d'un décalage entre la perception des spécialistes et celle de l'opinion publique. Pour le spécialiste, n'est il pas incongru de parler d'un secret entourant le 17 octobre? A ses yeux, le secret dénoncé par les média n'est il pas fantasmé?

Il est donc nécessaire d'analyser ce décalage entre spécialistes et opinion publique en tentant d'expliquer successivement les sentiments qui les animent. Enfin, une question concernant tous les chercheurs travaillant sur des sujets hâtivement qualifiés de "brûlants " se profile : quelle difficulté cette conjoncture crée-t-elle pour l'historien qui, aujourd'hui, veut travailler sur le 17 octobre?

\section{Un sentiment de " trop-plein "}

Les deux canaux de la connaissance historique que sont la bibliographie et les sources montrent que le 17 octobre est un événement relativement bien connu.

Le livre qui a fait rupture par son retentissement est celui de Jean-Luc Einaudi en 1991. Mais auparavant, en 1986, Michel Lévine avait publié Les ratonnades d'octobre aux éditions Ramsay. Cet ouvrage se fonde sur un recueil de témoignages et de documents présentés chronologiquement du 2 au 31 octobre 1961, avec des éclairages thématiques sur certains points d'histoire. C'est un livre riche en informations et pour tout travail entamé avant 1991, il représentait la principale référence sur le sujet. Aujourd'hui, l'oubli de ce livre est regrettable. Le problème qui se pose ici est de savoir pourquoi le livre de Jean-Luc Einaudi est apparu comme une révélation et pourquoi il est souvent présenté comme le premier ouvrage sur le 17 octobre 1961. 
La différence de portée entre les deux livres s'explique par le contexte au moment de leurs sorties respectives. Le livre de Jean-Luc Einaudi est sorti au moment de la commémoration du trentième " anniversaire " de l'événement, prise en charge par un collectif nommé "Au nom de la mémoire ". Ce collectif rassemblait des militants antiracistes et a pris de nombreuses initiatives pour réactiver le souvenir du 17 octobre : colloque, manifestation à Paris, réunions publiques diverses, réalisation d'un documentaire télévisé et édition d'un nouvel ouvrage appelé Le silence du fleuve, signé par Anne Tristan, livre militant, très riche en iconographie, visant un public très large. Certes, Jean-Luc Einaudi n'était pas membre de ce collectif et l'écriture de son livre, entreprise depuis plusieurs années, en était indépendante. Mais il se trouve que son livre est paru au moment où tout était fait pour médiatiser l'événement, ce qui a créé une conjoncture favorable à sa diffusion. Paradoxalement d'ailleurs, cette conjoncture lui a aussi été défavorable car le livre a été parfois perçu comme un livre militant au lieu de l'enquête qu'il est en réalité. Pour certains, le livre est alors apparu comme suspect a priori et il a pu être l'objet d'une lecture défavorable consistant à ne relever que les défauts du travail.

Le livre de Michel Lévine est donc resté dans l'ombre en raison de l'absence de résonance du 17 octobre dans l'opinion publique au moment de sa sortie. D'ailleurs, Michel Lévine n'aurait reçu que six lettres de lecteurs. Sa déception aurait été telle qu'il aurait jeté tous ses documents.

Pour l'historien, la bibliographie est à compléter par un livre privé de diffusion publique, en raison de sa saisie : Ratonnades à Paris, paru en 1961 même, chez Maspero. Paulette Péju, qui en est l'auteur, a principalement rassemblé des extraits de la presse. Seuls quelques exemplaires ont survécu, mais l'un d'entre eux se trouvant à la BDIC, ce livre est aujourd'hui facilement consultable. En ajoutant à cette liste le livre de Jean-Paul Brunet récemment paru, la bibliographie apparaît comme très hétéroclite, peut-être limitée car elle n'est constituée que de quelques livres, de qualités diverses. Mais le plus important est que l'ensemble de ces ouvrages fournit une base de connaissance sur le 17 octobre 1961 : I'événementiel de cette soirée est désormais connu. Et ce d'autant plus qu'il faudrait ajouter aux ouvrages publiés les travaux universitaires non diffusés par le canal de l'édition : plusieurs maîtrises, au moins trois, ont été consacrées au 17 octobre dans les années 1980-1990 ${ }^{3}$. Cette production sur le 17 octobre donne le sentiment que tout nouveau livre serait inutile, en tous cas sur le strict plan événementiel.

Par ailleurs, avant le travail de Jean-Paul Brunet se fondant principalement sur les archives de la préfecture de police, les chercheurs avaient contourné l'inaccessibilité des archives publiques en utilisant d'autres sources, au premier rang desquelles la presse $^{4}$. Les quotidiens métropolitains ont fait un récit de la soirée suffisamment détaillé pour connaître les principaux lieux de la manifestation, les conditions de sa répression et la violence déployée par les forces de l'ordre. Une lecture strictement événementielle des comptes rendus de ces quotidiens fait ainsi émerger un consensus sur certains points.

D'abord, I'Ouest parisien et le Quartier latin ont été les théâtres principaux de la manifestation et de sa répression. Ensuite, les policiers ont tiré sur les manifestants sans provocation de leur part, la passivité des cortèges étant relevée par tous les journalistes ${ }^{5}$. Cette passivité des manifestants a d'ailleurs suscité des interprétations diverses : pour certains, elle signale leur soumission au FLN. Les manifestants seraient là uniquement parce que le FLN les y a contraints. Elle est donc interprétée comme une docilité des manifestants qui subissent les événements sans en être acteurs. Pour d'autres, I'attitude des manifestants relève plus de la dignité que de la passivité. Enfin, tous les quotidiens font état d'arrestations massives, plus de 11000 manifestants ayant été interpellés sur un total de 20000 . Leur caractère raciste de ces arrestations au faciès est également reconnu, les exemples d'un touriste américain et d'un sénateur
3. Qu'on me permette de citer la mienne : La manifestation des A/gériens à Paris le 17 octobre 1961 et sa répression, sous la direction de Jean-Jacques Becker, soutenue en juin 1991. Disponible à la BDIC, cote F 4526 . 4. Cf. notre article « 17 octobre 1961 : une leçon pour l'écriture de I'histoire immédiate ". Cahier d'histoire immédiate $n^{\circ} 15$, printemps 1999, pp. 25-38. 5. Seule La Croix évoque des harkis "pris à partie" et "encerclés" au pont de Neuilly dans son édition du 19 octobre 1961. 
algérien membre de I'UNR malmenés par la police en attestant ${ }^{6}$.

La différence entre les quotidiens réside dans le jugement de valeur qu'ils portent sur la violence de la répression : nécessaire pour certains car il est inadmissible que l'adversaire occupe le pavé parisien, condamnable pour d'autres à cause de la passivité des manifestants. Par la suite, les comptes rendus des quotidiens sont alimentés par des témoignages et en particulier, par le courrier des lecteurs dont Le Monde signale qu'il est abondant.

Le plus intéressant est cependant le suivi de l'événement par la presse La plupart des journaux envoient un de leurs journalistes enquêter au bidonville de Nanterre, qui a fourni un très fort contingent de manifestants, ou à la Goutte d'Or. Ils y découvrent les conditions de vie des immigrés de la région parisienne ainsi que le poids de la répression à laquelle ils sont soumis par les contrôles d'identité, les vexations ou les brutalités de la police, conséquences du dispositif de lutte contre le FLN. Aux yeux de ces journalistes, les défilés du 17 octobre prennent alors une dimension sociale, les immigrés étant sortis des quartiers où ils sont habituellement confinés pour occuper l'espace parisien et rendre visible leur présence. Outre ces reportages dans les bidonvilles, la presse publie également des témoignages sur les centres où sont détenus les 11000 manifestants arrêtés. Ils émanent la plupart du temps de personnes chargées de la garde de ces centres ou appelées à y intervenir pour des raisons sanitaires et médicales. Tous rapportent l'existence de "violences à froid ", c'est-à-dire commises en dehors de la répression de la manifestation, et tous les condamnent. Enfin, plusieurs entrefilets signalent, à la fin du mois d'octobre, que des corps ont été retirés de la Seine et le cas de quelques Algériens, arrêtés et brutalisés par les forces de l'ordre, sont médiatisés, le plus célèbre étant Mohamed Badache. Ces exemples individuels se situent dans une période entourant le 17 octobre, pas forcément le soir du 17 octobre même, ce qui témoigne de l'insertion de la manifestation et de sa répression dans un contexte particulier, que la répression du 17 octobre permet de découvrir.

La presse fournit donc des informations nombreuses et variées avec - déjà — des questions d'interprétation sur la passivité des manifestants, leurs motivations, la légitimité de la répression et des pratiques policières, le contexte de cet automne $1961 .$.

En dehors de la presse et des témoignages qui ont pu être recueillis, les débats institutionnels permettent aussi d'approcher l'événement. A l'Assemblée nationale, au Sénat ou au Conseil municipal de Paris, le 17 octobre a fait irruption, soit au cours de séances qui lui ont été consacrées, soit au cours de débats concernant en théorie d'autres sujets. Les parlementaires ou les conseillers municipaux, qui n'appartiennent pas tous à l'opposition, se sont documentés pour pouvoir interroger le gouvernement. Outre une interprétation des événements, leurs interventions font aussi état des informations qu'ils ont recueillies par divers canaux. Enfin, les publications militantes se révè- lent utiles, en particulier une brochure syndicale, conçue par la CFTC, très bien implantée au sein de la police. Intitulée Face à la répression, d'une quinzaine de pages, elle est une mine d'informations sur les pratiques de la police dans la répression du nationalisme algérien avant même la manifestation, sur l'état d'esprit de la police, exaspérée par le terrorisme du FLN auquel elles est soumise, sur ses conditions de travail ou encore son recrutement ${ }^{7}$.

Cet ensemble de sources permet d'établir un certain nombre d'éléments concernant l'événementiel de la soirée et son contexte. Cette situation est tout à fait contradictoire avec le sentiment d'un " secret » autour de l'événement, d'autant plus que ces sources étaient accessibsles à l'époque même des faits. Comment expliquer dès lors l'idée qu'un secret couvre la soirée?

\section{Un sentiment de secret :}

L'existence d'un secret autour du 17 octobre relève des trois logiques précédemment exposées : le camouflage, l'oubli et l'occultation.

Le camouflage résulte de l'attitude des pouvoirs publics. Sur le moment, le gouvernement a tenté de bloquer autant que possible la circulation d'informations sur l'événement, comme en témoigne la saisie du livre de Paulette Péju.

Cette attitude pourrait s'expliquer par la volonté de nier les violences commises par la police. Le problème est qu'une telle négation manque totalement de crédibilité, la presse ayant donné les détails de la répression. C'est ainsi que Roger Frey oppose à Eugène Claudius-Petit, député du centre, qui l'interroge sur les violences à l'Assemblée nationale le 30 octobre, une réponse péremptoire qui se retourne contre lui : " Je n'ai pas eu entre les mains le début du commencement d'une ombre de preuve ». Son affirmation est si absurde comparée aux informations publiées par la presse que dès le lendemain, Libération, quotidien alors $d^{\prime}$ obédience communiste, titre en $1^{\text {re }}$ page : "M. Frey existe-t-il ? Pas le début du commencement d'une ombre de preuve ".

L'épisode est révélateur de l'inutilité pour le gouvernement de chercher à nier la violence de la répression. Dans ces conditions, les efforts qu'il déploie pour éviter toute enquête visent surtout à empêcher une analyse des responsabilités. En effet, Gaston Defferre, sénateur, plaide pour la formation d'une Commission d'enquête parlementaire. Le problème est qu'une ordonnance, en date du 17 novembre 1958, exclut la formation d'une telle Commission lorsque des informations judiciaires sont en cours sur les faits concernés. Utilisant ce texte, le gouvernement a fait ouvrir au Parquet de la Seine des informations judiciaires après le retrait de corps de "Nord-africains" de la Seine ou leur découverte sur la voie publique, en conséquence de quoi aucune Commission parlementaire ne put être nommée. Le gouvernement a donc bien manœuvré pour construire un secret, mais celuici vise plus les responsabilités que les faits en euxmêmes. L'argument ultime de Roger Frey contre la Commission proposée par Gaston Defferre, le 
14 décembre au Sénat, le prouve : il demande en effet "très instamment " aux sénateurs " de ne pas ajouter aux difficultés que nous connaissons en recréant les conditions mêmes d'un très lourd malaise qui est en voie d'apaisement et de règlement ". Le gouvernement n'hésite donc pas à arguer de sa crainte d'une agitation des milieux policiers qui lui seraient opposés. C'est que les rumeurs d'une infiltration de la police parisienne par l'OAS vont bon train à cette époque, contraignant le gouvernement à la ménager. D'ailleurs, le couvre-feu adopté le 6 octobre, détonateur de la manifestation du 17, l'a été après une rencontre entre un "Comité permanent de coordination et de défense de la police ", regroupant les trois principaux syndicats de policiers, et Maurice Papon. La base policière aurait-elle alors l'initiative sur ses supérieurs? Qu'ont fait ces derniers pour reprendre un pouvoir qui leur appartient légitimement? Le 17 octobre se fait ici l'excellent révélateur des rapports de force entre les différents secteurs du pouvoir métropolitain. En évitant l'analyse des responsabilités, le pouvoir politique couvre une police dont il semble redouter les réactions.

Hormis l'analyse des responsabilités, raison principale du voile jeté sur le 17 octobre par le gouvernement, l'absence d'enquête sur le 17 octobre a également produit ses effets sur certains aspects événementiels qui restent toujours méconnus ou difficiles à éclaircir. C'est le cas d'une rumeur faisant état d'un message radio sur les ondes de la police, selon lequel des policiers auraient été " éventrés à coups de couteau " par des manifestants ${ }^{8}$. L'existence d'un tel message pourrait permettre d'invoquer une manipulation de l'extrêmedroite pour expliquer la violence de la police. De même, un fait rapporté par Claude Bourdet est toujours resté sans réponse : dans la nuit du $17 \mathrm{au}$ 18 octobre, il a reçu la visite de policiers lui affirmant qu'une cinquantaine de manifestants ont été tués dans la cour de la préfecture de police. Conseiller municipal de Paris, Claude Bourdet a vainement interrogé Maurice Papon à ce sujet et, sans donner crédit à de tels témoignages non confirmés par ailleurs, la question se pose toujours de savoir ce qui s'est passé dans la cour de la préfecture de police de Paris le soir du 17 octobre. Cependant, les pages que Jean-Paul Brunet consacre dans son livre à ces aspects événementiels se révèlent peu probantes ${ }^{9}$. Ses recherches sont restées infructueuses. Mais la connaissance du 17 octobre tient-elle réellement à l'éclaircissement de tels aspects de la soirée? L'analyse y gagne-t-elle vraiment?

En effet, en comparaison, le bilan, lui aussi couvert par le secret, est une question centrale, d'une importance bien plus grande. Mais il est aujour$d^{\prime}$ hui très difficile à reconstituer alors qu'une enquête à l'époque même des faits aurait eu des chances de l'établir de façon relativement fiable, en ayant accès à tous les témoins aux dires facilement vérifiables.

II existe donc bien un secret entretenu par les pouvoirs publics sur le 17 octobre, couvrant principalement l'analyse des responsabilités et le bilan. Pour le protéger, les pouvoirs publics ont par la suite adopté une attitude de rétention d'informa- tions continue, en refusant systématiquement toute demande de dérogation pour la consultation des archives, jusqu'à une période très récente. Le plus remarquable est qu'aujourd'hui encore, l'évaluation des responsabilités et du bilan sont des questions liées I'une à l'autre, toujours dominantes, au cour des polémiques sur l'événement.

Deuxième mécanisme contribuant à plonger le 17 octobre dans un long coma, l'oubli, concernant la majorité de l'opinion publique, s'explique par l'attitude des Français à l'époque même des faits. N'ayant que peu ou pas d'intérêt pour l'événement, I'opinion l'a vite oublié. De façon ordinaire, seules des minorités militantes ont réagi et protesté : les sections parisiennes des grands syndicats - CGT, FO, CFTC, UNEF - adoptent une déclaration commune; un meeting a lieu à la Sorbonne; deux à trois mille personnes manifestent à l'appel du PSU le $1^{\text {er }}$ novembre $1961 . .$. Mais il ne se produit pas de réaction massive, ni de courant d'opinion en faveur des Algériens, contre la répression.

L'une des explications réside dans la stratégie du FLN qui n'a jamais été de rechercher le soutien de l'opinion publique française, considérée comme trop difficile à sensibiliser à la cause de l'indépendance algérienne. En faisant le choix du terrorisme, le FLN a du même coup renoncé au soutien de l'opinion. Dans le cas particulier du 17 octobre, la fédération de France aurait pu s'attendre à des réactions officielles de partis, des protestations larges car le caractère massif et pacifique de la manifestation corrige son image d'organisation groupusculaire et terroriste. Cependant, cette action ponctuelle n'est pas l'aboutissement d'un long travail, d'une longue stratégie, seule à même de sensibiliser l'opinion. De plus, la réceptivité de l'opinion à une telle stratégie se discute : les sondages montrent en effet que la paix est la valeur primant sur toutes les autres ${ }^{10}$. Or, une opinion pacifiste n'est pas forcément favorable à l'indépendance que revendique le FLN, même si, dans leur ensemble, les Français préféreraient voir l'Algérie se détacher de la France que de mourir pour elle.

Enfin, l'occultation concerne la minorité militante qui a réagi au 17 octobre avant de le gommer, de l'effacer de sa mémoire. Le mécanisme à l'œuvre est relativement simple : il s'agit de l'effacement du 17 octobre par la répression de Charonne. Pierre Vidal-Naquet en témoigne : le jour des obsèques des victimes de la répression de la manifestation au métro Charonne, un seul orateur a évoqué le 17 octobre, celui de la CFTC, le syndicat très implanté dans la police parisienne et engagé dans la dénonciation du 17 octobre ${ }^{11}$.

L'explication couramment avancée est que la répression de Charonne trouve plus aisément ses marques dans la mémoire collective que celle du 17 octobre 61 car, à Charonne, les manifestants sont des militants de la gauche française. La répression de Charonne évoque donc facilement une tradition de manifestation et d'affrontement du " peuple parisien " avec les forces de l'ordre qui le répriment durement. Cette tradition de la culture politique française remonterait à la Commune ${ }^{12}$. De plus, la réaction de protestation contre
8. D'après Le Monde du 14 novembre 1961 , p. 2. 9. Jean-Paul Brunet a retrouvé la trace d'un message radio provocateur dans les archives, mais d'une teneur différente. Cf. Police contre FLN, Le drame d'octobre 1961, Paris, Flammarion, 1999, pp. 183-185. Sur la cour de la préfecture de police : cf. pp. 228-230. 10. Cf. l'étude pionnière de Charles-Robert Ageron, "L'opinion française à travers les sondages ", in $L a$ guerre d'Algérie et les Français, sous la direction de Jean-Pierre Rioux, Paris, Fayard, 1990, pp. 25-44.

11. Cf. "Ce jour qui n'ébranla pas Paris " in Face à la raison d'Etat, Paris, La Découverte, 1989 , pp. 162-166. 12. Cf. les analyses d'Isabelle Lambert "Vingt ans après " et de Robert Frank "Les troubles de la mémoire française ", in La guerre d'Algérie et les Français, op. cit., respectivement $p p$. 553-559 et pp. 603-607. 
Au soir de la manifestation du 17 octobre 1961. Photographie d'Elie Kagan, dont le Musée d'Histoire Contemporaine - BDIC vient de récupérer l'ensemble des archives photographiques (plus de trois cent mille négatifs originaux). (Coll. $M H C-B D I C)$
13. Interview accordée au journal Le Matin, le 17 octobre 1985, p. 3. la répression de Charonne suscite la fierté puisqu'elle s'est soldée par une grande manifestation de 500000 personnes.

Au contraire, le souvenir du 17 octobre est culpabilisant pour deux raisons : I'indifférence générale qui a suivi la répression est une attitude qui ne suscite en rien la fierté; de plus, la nature de la répression renvoie dans la mémoire collective à d'autres moments de " honte nationale ". L'analogie entre les arrestations massives le soir du 17 octobre et les rafles de Juifs pendant la Seconde Guerre mondiale est en effet très courante dans les journaux de l'époque. C'est donc une répression lourde à assumer. Il est d'ailleurs révélateur qu'aujourd'hui, le 17 octobre resurgisse à travers la Seconde Guerre mondiale, à l'occasion de l'étude des responsabilités, de la culpabilités des Français durant cette période.

Ces conditions de camouflage, d'oubli et d'occultation expliquent le sentiment qu'un secret entoure le 17 octobre et qu'il doit être percé. Le problème qui se pose à ce stade est de savoir, dans ces conditions, comment les générations des années 1980-1990 ont pu redécouvrir l'événement. Autrement dit : dans quels secteurs de l'opinion le souvenir du 17 octobre est-il resté assez vif pour resurgir trente ans plus tard?

II s'agit en fait de l'immigration et pas seulement algérienne. Les immigrés des bidonvilles de I'Ouest parisien - Nanterre, Gennevilliers, Argenteuil... - venaient en effet de tout le Maghreb et tous ont été témoins de la manifestation ainsi que de sa répression. Tous ont connu une personne qui est allée manifestée, voire qui a été frappée, blessée, arrêtée ou même qui n'est pas revenue. Les générations des années 1980 - 1990 qui redécouvrent le 17 octobre sont celles de leurs enfants, confrontés à la montée du Front national. Dans ce contexte en effet, se forment et se développent des organisations antiracistes qui multiplient leurs activités. Ce sont ces organisations, au sein desquelles des enfants d'immigrés sont engagés, qui ont remis le 17 octobre sur la place publique. Le principal animateur du collectif "Au nom de la mémoire " est ainsi le fils d'un immigré algérien de Bezons, commune limitrophe de Nanterre, dirigeant d'une association antiraciste. II a entendu parler du 17 octobre par des anciens du FLN et par son propre père. Quant à Anne Tristan, auteur du livre Le silence du fleuve, édité par ce collectif, elle avait précédemment rédigé $A u$ front, un livre dénonçant le Front national, dans lequel elle raconte son infiltration du parti d'extrême-droite à Marseille.

Pour les militants antiracistes des années 1980 , le 17 octobre est doté d'une forte valeur pédagogique car il est l'exemple à ne pas suivre. C'est ce qu'explique Harlem Désir, fondateur de SOS Racisme, à propos d'une manifestation de commémoration du 17 octobre : "pouvoir comprendre ce qui s'est passé, c'est travailler à ce que cela ne se reproduise pas" $"{ }^{13}$.

Ces associations ou personnalités, porteuses de mémoire, vecteurs de mémoire, tiennent le discours de la révélation de l'événement, à chacune de leurs initiatives, car elles se heurtent, en face, au camouflage des pouvoirs publics, à l'oubli de l'opinion, à l'occultation chez certains, qu'elles veulent combattre. Ce discours de la révélation alimente l'idée qu'il existe un secret alors même qu'aujourd'hui il existe des livres, des documents qui permettent de connaitre le 17 octobre. C'est ici que naît le sentiment d'un fantasme du secret autour de l'événement. L'historien qui travaille sur le 17 octobre est donc placé dans une conjoncture particulièrement difficile : comment envisager sous tous les angles problématiques possibles l'histoire d'un événement totalement dominé par une exigence de vérité et de transparence sur le nombre des victimes? 


\section{Quelle difficulté pour l'historien?}

Le problème principal est que le questionnement de l'historien sur le 17 octobre est piégé par cette conjoncture. En effet, le secret entourant le 17 octobre couvrant essentiellement le bilan et I'analyse des responsabilités, l'historien a le devoir de s'attacher au dénombrement des morts, dont dépendrait ensuite l'évaluation des responsabilités.

Le risque est ici de se laisser dicter sa problématique par le contexte. En effet, les militants qui ont fait resurgir le 17 octobre se fondent sur un bilan fixé aux environs de deux cents morts par Jean-Luc Einaudi dans La bataille de Paris et parlent d'un " massacre ", de la responsabilité voire de la culpabilité de Maurice Papon. Le danger qui guette I'historien est de vouloir répondre à ces affirmations et, du même coup, restreindre son champ d'analyse à ces deux questions : y a-t-il vraiment eu deux cents morts? Maurice Papon en est-il responsable? Le risque pour l'historien, même s'il se défend d'être lui-même un militant, est de finir par s'enfermer dans une problématique qui devient elle-même militante et qui consiste à défendre la thèse strictement opposée : le nombre de morts n'est pas de deux cents et la responsabilité de Maurice Papon n'est pas établie. La démarche est intellectuellement stérile car elle conduit à l'affrontement de deux thèses irréductibles, situation peu productive sur le plan de la progression de la connaissance et de la vérité qui, souvent, combine les hypothèses les plus contradictoires. Mais surtout : elle appauvrit le champ des recherches historiques sur le 17 octobre en réduisant le questionnement au seul nombre de morts.

En réalité, les problématiques ouvertes par cette manifestation sont multiples et elles peuvent s'élargir à des questionnements qui dépassent cette seule journée. Ici est la richesse de cet événement, l'avenir d'éventuelles recherches fructueuses se fondant sur des fonds d'archives très divers.

La première question est de savoir si, malgré l'absence de provocation de la part des manifestants, le FLN porte une responsabilité dans la répression. Autrement dit, la fédération de France du FLN a-t-elle cherché l'affrontement avec la police simplement en lançant un mot d'ordre de manifestation alors qu'elle connaissait l'état d'exaspération de la police parisienne causé par les attentats? Replacée dans le contexte des négociations en cours, une telle hypothèse suppose que la fédération de France a cherché à relancer l'affrontement car elle était opposée aux négociations. Dans ce cas, elle aurait été opposée au GPRA qui conduisait ces négociations. Cette piste mène à I'histoire interne du FLN qu'il faut interroger. En dehors d'un éventuel désaccord sur la conduite des négociations, la manifestation du 17 octobre exprime-t-elle des tensions au sein du FLN? Tout le monde était-il d'accord avec cette décision? Comme cela a parfois été suggéré, la décision d'organiser la manifestation a-t-elle relevé des seuls représentants parisiens de la fédération de France du FLN, en désaccord avec son Comité directeur? Ces désaccords préparent-ils des affrontements en vue de la prise du pouvoir après l'in- dépendance? La manifestation prend une dimension politique nouvelle : elle n'est pas qu'un affrontement " police contre FLN", elle est aussi un enjeu entre factions internes du FLN. L'histoire du 17 octobre ne sera connue que lorsque cette question aura été élucidée.

La deuxième problématique relève de l'histoire sociale et non de l'histoire politique. Il s'agit de se demander, étant donné les conditions de vie des immigrés algériens dans les bidonvilles et les contrôles policiers, voire les brutalités policières qu'ils connaissent, s'ils n'avaient pas leurs propres motivations pour descendre dans la rue ce soir-là. Ou ne sont-ils réellement descendus dans la rue que sous la menace du FLN ? II faut interroger ici I'histoire sociale et faire au moins un tableau de l'immigration algérienne en cet automne 1961 : combien sont-ils? Où vivent-ils? Comment viventils? Dans quelle mesure la répression censée toucher le FLN les atteint-elle? De ce point de vue, le principal défaut du livre de Jean-Paul Brunet est précisément son titre Police contre $F L N$, laissant de côté les 20000 manifestants, alors que se pencher sur leur sort ouvre la réflexion sur la nature de la manifestation : est-elle uniquement une manifestation politique s'inscrivant dans le cadre $d^{\prime} u n$ affrontement entre la France et le FLN, voire d'affrontements internes au nationalisme algérien, ou est-elle aussi une manifestation sociale de démonstration par les immigrés de leur présence sur le sol français? Ou encore : est-elle tout à la fois? Beaucoup d'observateurs ont noté l'apparente dignité des manifestants le soir du 17 octobre, le fait qu'ils étaient endimanchés - acte d'une signification réelle à cette époque-, le fait qu'il était très inhabituel de les voir dans les beaux quartiers de la capitale. D'ailleurs, à ce propos, le terme de manifestation ne semble pas approprié pour désigner la présence de ces 20000 personnes dans les rues de Paris ce soir-là. Il faut redescendre à un niveau purement événementiel : l'ordre donné par le FLN est avant tout de boycotter un couvre-feu, de descendre dans la rue, de se promener sur les grandes artères parisiennes pour ignorer la mesure préfectorale. La manifestation n'en est plus une : elle est une démonstration collective. Sous cet angle, tout immigré, pas seulement les Algériens mais tous ceux pour qui le couvre-feu est source d'ennuis, pouvait avoir une motivation pour obéir au mot d'ordre lancé par le FLN. Cette simple probabilité impose de ne pas s'en tenir à l'explication de la contrainte pour comprendre le succès de la manifestation.

Enfin, la dernière problématique concerne évidemment le bilan qui, s'il ne doit pas être l'unique question guidant le travail, n'en est pas pour autant une question à ignorer. Le plus étonnant est que le bilan lui-même ouvre la voie à des interrogations qui dépassent largement le seul dénombrement des victimes. En effet, aujourd'hui, le dépouillement des archives publiques permet d'établir le nombre des victimes à environ une quarantaine : Jean-Paul Brunet dans son livre, ainsi que Jean Géronimi dans son rapport, parviennent à ce résultat. Quant à l'évaluation ancienne de deux cents morts, elle s'explique : il s'agit d'une évaluation qui prend en compte des personnes décé- 
14. In La bataille de Paris, Paris, Seuil, 1991, pp. 313-317.

15. In Police contre FLN le drame d'octobre 1961 Paris, Flammarion, 1999, p. 138. Jean-Paul Brunet précise que sur ces 308 cas, 60 sont " douteux" c'est-à-dire qu'il n'est pas certain que le décès résulte d'un homicide. Pour les seuls mois de septembre et octobre, le nombre de " cas

douteux " est de 34 . Le total pourrait donc être ramené à 248 pour toute l'année 1961, dont 107 au cours des seuls mois de septembre et octobre. 16. D'après les déclarations de Roger Frey à l'Assemblée nationale, le 13 octobre 1961 . Une lo de finances rectificative permis la création de 300 emplois supplémentaires dans la police parisienne en juillet 1961.

17. In Police contre FLN, le drame d'octobre 1961 op. cit., pp. 133-134. dées avant le 17 octobre, voire aussi après. La liste de personnes tuées publiées par Jean-Luc Einaudi en annexe de son livre prend ainsi en compte des personnes tuées bien avant le 17 octobre $^{14}$. Les décomptes faits par Jean-Paul Brunet aboutissent également à ce constat : sur toute l'année 1961, 308 cadavres de "Nord-Africains " morts par homicide sont entrés à l'Institut médico-légal, dont 141 durant les seuls mois de septembre et octobre $1961^{15}$.

Cette situation interpelle car elle signifie que toute tentative pour évaluer le nombre de morts de la seule soirée du 17 octobre débouche sur la découverte d'une période particulière : pourquoi autant de décès au cours des mois de septembre et d'octobre? Que s'est il passé durant l'été et l'automne 1961 en métropole et en particulier dans la région parisienne? Finalement la question du bilan n'est plus une simple question de dénombrement. Elle ouvre la voie à l'étude d'un contexte. Elle incite à élargir les recherches à l'été et l'automne 1961, période qui semble avoir été meurtrière. Le contexte est en effet celui d'un redoublement des attentats du FLN à la fin du mois d'août. Du côté policier, les effectifs de la police parisienne ont été renforcés à la fin du mois de juillet ${ }^{16}$. Jean-Paul Brunet révèle par ailleurs l'existence de groupe " para-policiers " qu'il définit ainsi : " nous entendons par là des groupes, soit de policiers qui n'étaient pas en service, soit d'amis politiquement très proches $d^{\prime}$ eux, et qui enlevaient des Algériens ou les recevaient des mains de patrouilles de policiers, pour aller "se faire justice eux-mêmes " " 17 .
Alors, pourquoi un tel pic d'affrontement alors que des négociations sont en préparation? Cet été et cet automne 1961 sont-ils marqués par des tentatives pour relancer l'affrontement et torpiller tout espoir de négociations de la part de ceux qui les refusent? Du côté français, un fait donne à réfléchir : Edmond Michelet, ministre de la Justice du général de Gaulle, représentant la tendance libérale du gouvernement, démissionne en août. Simple coïncidence? Signe d'un raidissement de la politique gouvernementale?

S'interroger sur le bilan n'est donc pas seulement compter les morts et confronter deux thèses à départager mais comprendre d'où viennent les évaluations et finalement, réfléchir sur le contexte entourant la manifestation, découvrir cette période particulière de la guerre d'Algérie en France, dont I'histoire reste à écrire.

Plus largement, cet exemple amène à une question plus vaste : le rôle de l'historien est-i.l de percer les secrets et de répondre à une exigence de vérité ? Dans une certaine conception, peutêtre. En tous cas, percer le secret ne doit pas être son unique objectif, faute de quoi le risque existe de se laisser piéger, emporter par une conjoncture fiévreuse entourant l'objet de son travail, situation relativement courante pour des sujets appartenant à "l'histoire de notre temps».

Sylvie THÉNAULT Docteur en histoire 